\title{
Mid-Infrared Enhanced Lenticulars caught in the dusty midst of transformation in the Coma Cluster
}

\author{
Laurie Riguccini ${ }^{1,2}$, and Pasquale Temi ${ }^{3}$, and Alexandre Amblard ${ }^{3}$, \\ and Michael Fanelli ${ }^{3}$, et al. \\ ${ }^{1}$ Observatório do Valongo, UFRJ, Ladeira do Pedro Antônio 43, Saúde, \\ Rio de Janeiro, RJ 20080-090, Brazil (riguccini@astro.ufrj.br) \\ ${ }^{2}$ CAPES/BJT Science Without Borders Postdoctoral Fellow, Brazil \\ ${ }^{3}$ NASA Ames Research Center, Moffett Field, CA, United States.
}

\begin{abstract}
We explore the properties of early-type galaxies (ETGs) in rich environments such as clusters of galaxies. The $\mathrm{L}_{24} / \mathrm{L}_{K}$ distribution of ETGs in both Virgo and Coma clusters shows that some lenticulars (S0, 10 in Coma and 3 in Virgo) have a much larger $\mathrm{L}_{24} / \mathrm{L}_{K}$ ratio $(0.5$ to $\sim 2$ dex) than the bulk of the ETG population. We call these sources Mid-Infrared Enhanced Galaxies (MIEGs). In Coma, they are mostly located in the South-West part of the cluster where a substructure is falling onto the main cluster. MIEGs present lower g-r color than the rest of the ETGs, because of a blue continuum. We interpret the excess $\mathrm{L}_{24} / \mathrm{L}_{K}$ ratio as evidence for an enhanced star-formation induced as a consequence of their infall into the main cluster.
\end{abstract}

Keywords. galaxies: elliptical and lenticular, cD, galaxies: clusters: individual (Virgo, Coma)

\section{Introduction}

Studies in the past decades have highlighted differences in the ETG stellar population in dense clusters and in lower density environments, based on spectral line ratios in these galaxies (e.g., Bower et al.1990). Caldwell et al. (1993) found similar evidence, arguing that a basic visual inspection of ETG spectra is already enough to distinguish the stellar populations of ETGs lying in distinct environments.

A tight correlation between the $24 \mu \mathrm{m}$ luminosity and the K-band luminosity is expected for ellipticals (E), where most of the $24 \mu \mathrm{m}$ emission is circumstellar in origin (e.g., Temi et al.2009). They found a larger scatter in this relation for S0 when compared to E, and interpreted it as the signature of a subpopulation of younger stars in some S0. We investigate the effect of the environment on the $\mathrm{L}_{24} / \mathrm{L}_{K}$ ratios of ETGs studying the Virgo and Coma ETGs populations (see also Riguccini et al.2015).

\section{Main conclusions}

Recently, a population of S0s with signs of enhanced star formation according to their high MIR/NIR fluxes (Fig. 1a) has been identified in Virgo and Coma (Mid-IR Enhanced Galaxies or MIEGs, Riguccini et al.2015). MIEGs exhibit diverse morphologies, from globally, asymmetric systems to redder, more relaxed galaxies. The majority of the MIEGs in Coma (7 sources among the 10 ones, cf dashed ellipse on Fig. 1b) are located in the southwest part of Coma, known for an ongoing merger activity. MIEGs also have bluer g-r color than the rest of the ETG population, with g-r colors similar to late-type galaxies. Their spectra are different from typical ETG spectrum, and feature strong emission lines (e.g., $\mathrm{H}_{\alpha}, \mathrm{H}_{\delta}, \mathrm{OII}$ ) that are good indicators of star-formation. Three ETGs in Coma present similar g-r colors than some of the MIEGs, they are bluer than the bulk of the ETG population because of their blue continuum. They do not show any strong emission lines that could account for star-formation activity like MIEGs. However these "blue sources" are all located in the neighborhood of MIEGs (Fig. 1b) that hint toward a common history and a common evolution different from the rest of the ETG population. 

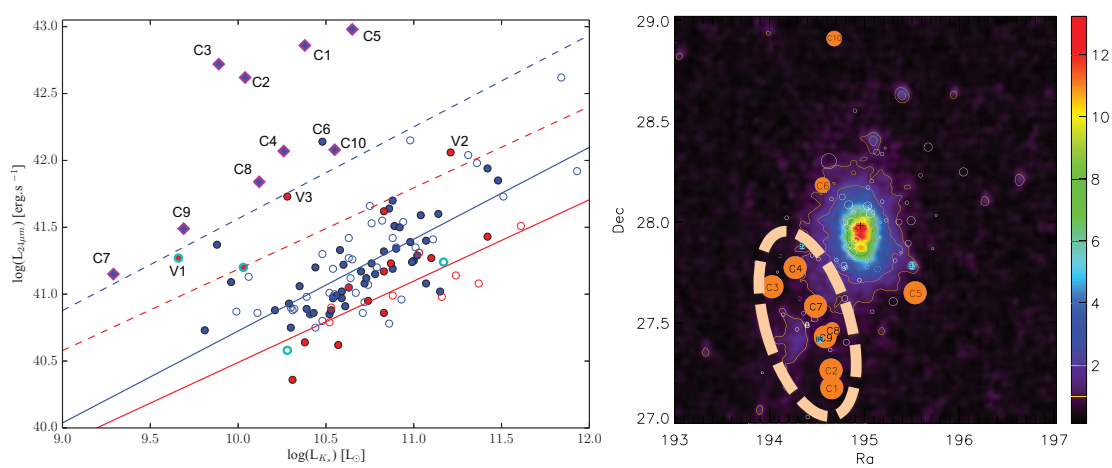

Figure 1. Left: $24 \mu \mathrm{m}$ luminosity as a function of the K-band luminosity in logarithmic scale for ETGs in the Coma (in blue) and Virgo (in red) clusters. The dashed lines represent the MIEG selection line for Coma (blue) and Virgo (red) galaxies. Right: Distribution of the $24 \mu \mathrm{m}$ detected sources on top of the ROSAT map of the Coma cluster: white open circles, orange (MIEGs) and blue ("blue ETGs") filled ones. The size of the circle is proportional to the ratio $\mathrm{L}_{24 \mu m} / \mathrm{L}_{K}$ (i.e proportional to the specific star-formation rate of the source).

These results emphasize the potential link between cluster merger activity and star-formation in galaxies. From a mid-to-near IR analysis, we have selected transiting galaxies at different stages of their evolutionary history. We have selected ETGs with signs of strong star formation and a subclass of ETGs with blue optical colors but no remaining of current star formation. MIEGs are a peculiar population of S0 galaxies with signs of enhanced star formation (high $\mathrm{L}_{24 \mu m} / \mathrm{L}_{K}$ ratios, strong emission lines, blue optical colors) potentially located in cluster substructure merging area. The known ongoing merger activity of the Coma region, where most of Coma MIEGs are located, could account for this enhancement. The hypothesis of cluster merging triggering the star formation has been discussed (e.g., Gavazzi et al.2003), mostly for starbursts. In the other hand, the blue sources discussed in this work are good post-MIEG candidates where the recent star formation would have been quenched due to ram pressure stripping (e.g., Baldi et al.2001). These blue sources would be the analogs of the post-starburst candidates in an evolutionary path for ETGs where MIEGs would be the analog of starburst galaxies. We will focus in the near future on the formation of these MIEGs, analyzing for instance their stellar population and looking for a second burst of star formation.

\section{References}

Baldi, A., Bardelli, S., \& Zucca, E. 2001, MNRAS, 324, 509

Bower, R. G., Ellis, R. S., Rose, J. A., \& Sharples, R. M. 1990, AJ, 99, 530

Caldwell, N., Rose, J. A., Sharples, R. M., Ellis, R. S., \& Bower, R. G. 1993, AJ, 106, 473

Gavazzi, G., Cortese, L., Boselli, A., Iglesias-Paramo, J., Vlchez, J. M., \& Carrasco, L. 2003, ApJ, 597, 210

Riguccini, L., Temi, P., Amblard, A., Fanelli, M., \& Brighenti, F. 2015, ApJ, 810, 138

Temi, P., Brighenti, F., \& Mathews, W. G. 2009, ApJ, 707, 890 